\title{
Progress toward standardized diagnosis of vascular cognitive impairment: Guidelines from the Vascular Impairment of Cognition Classification Consensus Study
}

\author{
Olivia A. Skrobot ${ }^{\mathrm{a}}$, Sandra E. Black ${ }^{\mathrm{b}}$, Christopher Chen ${ }^{\mathrm{c}}$, Charles DeCarli ${ }^{\mathrm{d}}$, Timo Erkinjuntti ${ }^{\mathrm{e}}$, \\ Gary A. Ford ${ }^{\mathrm{f}}$, Rajesh N. Kalaria ${ }^{\mathrm{g}}$, John O’Brien ${ }^{\mathrm{h}}$, Leonardo Pantoni ${ }^{\mathrm{i}}$, Florence Pasquier ${ }^{\mathrm{j}}$, \\ Gustavo C. Roman ${ }^{\mathrm{k}}$, Anders Wallin, ${ }^{1, \mathrm{~m}}$, Perminder Sachdev ${ }^{\mathrm{n}, \mathrm{o}}$, Ingmar Skoog ${ }^{\mathrm{m}}$, \\ the VICCCS group ${ }^{1}$, Yoav Ben-Shlomo ${ }^{p}$, Anthony P. Passmore ${ }^{\mathrm{q}}$, Seth Love ${ }^{\mathrm{a}}$, Patrick G. Kehoe ${ }^{\mathrm{a}, *}$ \\ ${ }^{a}$ Translational Health Sciences, University of Bristol, Bristol, UK \\ ${ }^{b}$ Sunnybrook Research Institute, University of Toronto, Ontario, Canada \\ ${ }^{c}$ Department of Pharmacology, National University of Singapore, Singapore \\ ${ }^{d}$ Department of Neurology and Center for Neuroscience, University of California at Davis, Sacramento, California, USA \\ ${ }^{e}$ Neurology, University of Helsinki and Helsinki University Hospital, Helsinki, Finland \\ ${ }^{f}$ Divison of Medical Sciences, Oxford University, Oxford, UK \\ ${ }^{g}$ Institute of Neuroscience, Newcastle University, Newcastle, UK \\ ${ }^{h}$ Department of Psychiatry, University of Cambridge School of Clinical Medicine, Cambridge, UK \\ ${ }^{i}$ NEUROFARBA Department, University of Florence, Florence, Italy \\ ${ }^{j}$ Inserm U1171, University of Lille, Lille, France \\ ${ }^{k}$ Methodist Neurological Institute, Houston, Texas, USA \\ ${ }^{l}$ Memory Clinic at Department of Neuropsychiatry, Sahlgrenska University Hospital, Mölndal, Sweden \\ ${ }^{m}$ Institute of Neuroscience and Physiology, Sahlgrenska Academy, University of Gothenburg, Sweden \\ ${ }^{n}$ School of Psychiatry, University of New South Wales, Sydney, Australia \\ ${ }^{o}$ Neuropsychiatric Institute, Prince of Wales Hospital, Randwick, New South Wales, Australia \\ ${ }^{p}$ Population Health Sciences, University of Bristol, Bristol, UK \\ ${ }^{q}$ Institute of Clinical Sciences, Block B, Queens University Belfast, Belfast, UK
}

Abstract Introduction: Progress in understanding and management of vascular cognitive impairment (VCI)
has been hampered by lack of consensus on diagnosis, reflecting the use of multiple different assess-
ment protocols. A large multinational group of clinicians and researchers participated in a two-phase
Vascular Impairment of Cognition Classification Consensus Study (VICCCS) to agree on principles
(VICCCS-1) and protocols (VICCCS-2) for diagnosis of VCI. We present VICCCS-2.
Methods: We used VICCCS-1 principles and published diagnostic guidelines as points of reference
for an online Delphi survey aimed at achieving consensus on clinical diagnosis of VCI.
Results: Six survey rounds comprising 65-79 participants agreed guidelines for diagnosis of VICCCS-
revised mild and major forms of VCI and endorsed the National Institute of Neurological Disorders-Ca-
nadian Stroke Network neuropsychological assessment protocols and recommendations for imaging.
Discussion: The VICCCS-2 suggests standardized use of the National Institute of Neurological
Disorders-Canadian Stroke Network recommendations on neuropsychological and imaging assess-
ment for diagnosis of VCI so as to promote research collaboration.

(C) 2017 the Alzheimer's Association. Published by Elsevier Inc. All rights reserved.

\footnotetext{
${ }^{1}$ Members are listed at the end of the article.

*Corresponding author. Tel.: 0117414 7821; Fax: 01174147822
} 


\section{Introduction}

Since Hachinski et al [1] proposed the term multi-infarct dementia to describe dementia complicating ischemic vascular disease, numerous other descriptors have been used to encompass the heterogeneous clinical and etiological spectrum of cognitive impairment due to cerebrovascular disease (CVD). These include vascular dementia (VaD), vascular cognitive impairment (VCI), subcortical (ischemic) vascular dementia, and vascular cognitive disorders (VCDs), variably diagnosed according to multiple different guidelines or protocols [2-14], some agreed by national institutions or research networks, for example, Alzheimer's Disease Diagnostic and Treatment Centers [11], International Statistical Classification of Diseases, 10th revision [15], the National Institute of Neurological Disorders and Stroke (NINDS)-Association Internationale pour la Recherche et l'Enseignement en Neurosciences [16], and Diagnostic and Statistical Manual of Mental Disorders, fourth and fifth editions (DSM-4 and DSM-5; [17,18]).

Studies comparing some of these protocols have shown they are not readily interchangeable [19-21]. After the commencement of the Vascular Impairment of Cognition Classification Consensus Study phase 1 (VICCCS-1), the American Heart Association/American Stroke Association (AHA/ASA) published a statement on vascular contributions to cognitive impairment and dementia [22]. This supported the use of assessment protocols previously published by NINDS-Canadian Stroke Network (CSN) [13]. There have been other recent contributions to this field from the International Society of Vascular Behavioral and Cognitive Disorders (VASCOG) [23] and the DSM-5 [18]. The level of take up of these recent guidelines is still unclear. Only those published during VICCCS-1, before commencement of VICCCS-2, could be included for consideration in the present study $[22,24]$.

The aim of VICCCS was to achieve broad international consensus on diagnosis of VCI, through participation of a large pool of international researchers and clinicians in an iterative survey using the Delphi approach. After two initial survey rounds, the study was separated into two phases: VICCCS-1, addressing key concepts in our understanding and terminology of cognitive impairment resulting from CVD [25], and VICCCS-2, focusing on the formulation of practical guidelines for diagnosis.

The VICCCS-1 achieved broad consensus on concepts of VCI. It supported the use of "mild" and "major" subdivisions of the severity of impairment, aligning with the revised terminology in the DSM-5. VICCCS-1 participants concluded that attempts to separate mild VCI into further subtypes according to affected cognitive domains were at present premature but agreed that this should be an area of future research. VICCCS-1 agreed (Fig. 1, reproduced from [25]) that the major forms of VCI $(\mathrm{VaD})$ should be classified into four main subtypes: (i) post-stroke dementia (PSD); (ii) subcortical ischemic vascular dementia (SIVaD); (iii) multi-infarct (cortical) dementia (MID); and (iv) mixed dementias (further subdivided according to additional neurodegenerative pathologies). Framed by these concepts, VICCCS-2 used the same Delphi methodology to agree diagnostic guidelines on determination of severity of VCI and discrimination of subtypes.

\section{Methods}

Participants in VICCCS-1 [25] were invited to participate in VICCCS-2 (Supplementary Fig. 1). Although 149 initially agreed to participate, only approximately half were active and committed respondents in three or more rounds, with low attrition and little variation in participation throughout the six rounds (65-79 participants in each round, a mean of 72). Of the active participants, $63 \%-75 \%$ of participants (mean 68\%) were clinicians with direct involvement in clinical assessment or health service patient care. The remainder were nonclinical (i.e., supporting clinical work technically or otherwise, but not involved in clinical decision-making, or predominantly involved in research). Individual round representation is provided in Supplementary Table 1.

\subsection{Data collection}

We used the Delphi method, an iterative, multistaged series of structured questionnaires with feedback of anonymized responses and progressive refinement of questions to reach consensus [26]. The process was co-ordinated by a nonparticipating researcher (O.A.S). Anonymization of responses facilitated free expression of opinion throughout the study. Feedback of summary responses after each round informed subsequent questions and allowed unbiased evolution of group judgment. A threshold of two-thirds agreement was chosen to signify consensus [27] for issues refined iteratively through multiple rounds, as in VICCCS-1 [25]. For issues where this threshold was not reached, we present the summary data including the view that was most strongly supported. A summary of topics covered in each Delphi round is presented in Fig. 2. The first two rounds were used to select from previous publications those diagnostic criteria deemed most valuable as a basis for further discussion. These provided main themes addressed in the four subsequent rounds (November 2012-September 2013). Consensus views from topics addressed in VICCCS-1 were used in discussions.

\section{Results}

\subsection{VICCCS foundation rounds and VICCCS-2 rounds 1 and 2}

In the two foundation rounds of VICCCS, the consensus view ( $94 \%$ of respondents) was that none of the current diagnostic protocols was fully fit for purpose and that formulation of improved assessment criteria for VCI was a 


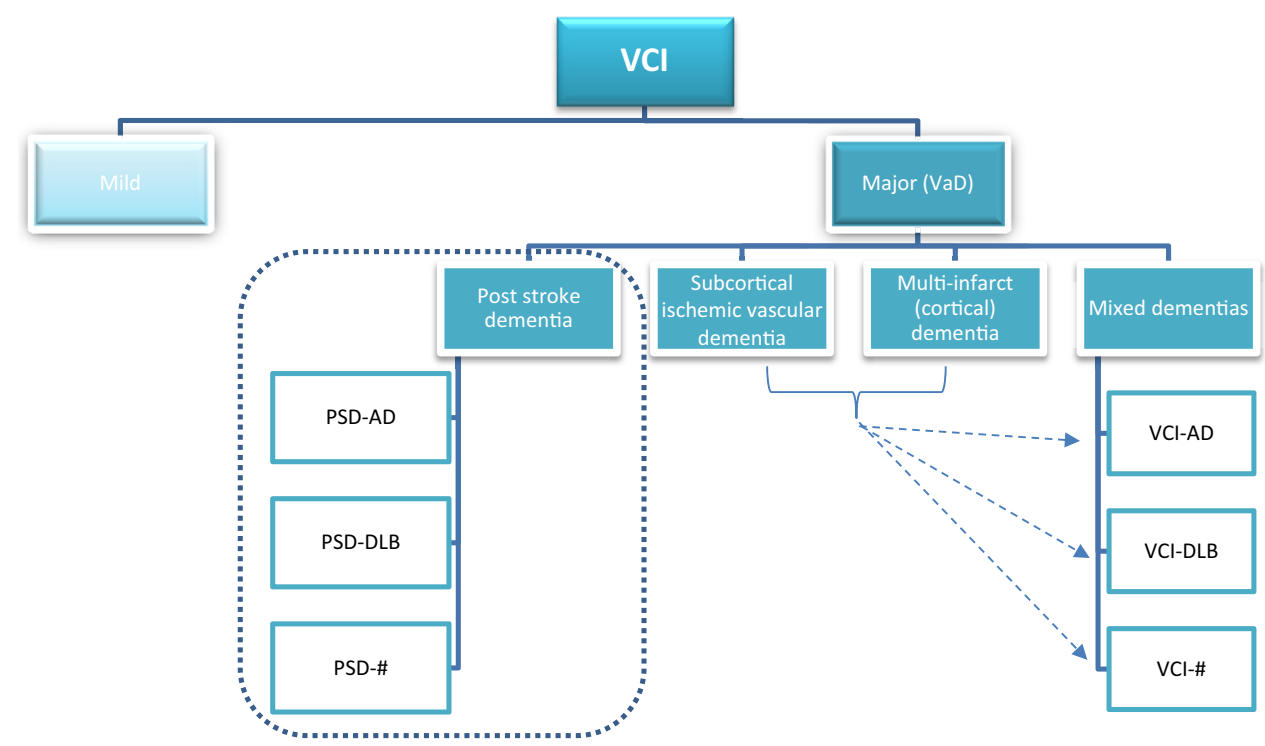

Fig. 1. Revised conceptualization of VCI in VICCCS. Subtypes of VCI are divided according to level of VCI into mild VCI and major VCI (VaD). Mild VCI is not further subdivided at this time. Major VCI $(\mathrm{VaD})$ is classified into four main subtypes as depicted. The 6-month temporal basis (denoted by the dashed box) for cognitive decline after stroke differentiates post-stroke dementia (PSD) from other forms of major VCI (VaD). PSD and mixed dementias are further delineated if a comorbid neuropathology is present (AD and DLB are given as examples, with \# denoting other possible combinations). Subcortical ischemic vascular dementia or MID subtype cases were with these specific types of dementia alone; however cases also presenting with any other neurodegenerative pathology would then be categorized as mixed dementias (dashed arrows) according to the comorbidities present. Abbreviations: AD, Alzheimer's disease; DLB, dementia with Lewy bodies; MID, multi-infarct (cortical) dementia; PSD, post-stroke dementia; VaD, vascular dementia; VCI, vascular cognitive impairment; VICCCS, Vascular Impairment of Cognition Classification Consensus Study.

priority. Data from the foundation rounds, including the researcher-led [2,6,7,11-14,16,28-30] and organizationbased diagnostic criteria/protocols that were critiqued by the participants, are provided as Supplementary Material.

Participants selected their preferred criteria of the six presented (four were chosen as the best starting points in VICCCS foundation rounds $[6,12,13,31]$ and two that were published during VICCCS-1 could therefore be critiqued in VICCCS-2 $[22,24])$. Of these six, the AHA/ASA Scientific Statement: vascular contributions to cognitive impairment and dementia [22], henceforth referred to as AHA/ASA, was the first preference of the highest proportion of respondents $(41 \%)$, followed by NINDS-CSN [13] (25\%). The AHA/ASA does not provide assessment protocols but refers to the recommendations made in the NINDS-CSN. These guidelines provided the basis for further discussion and elaboration.

According to most respondents (65\%), VICCCS-2 aimed to provide a single set of diagnostic guidelines for clinical and research use. Agreed objectives were to develop a clear and efficient protocol that was simple to use and would yield readily interpretable results, allowing discrimination of VCI subtypes and severity.

\subsection{VICCCS-2 rounds 3-6}

\subsubsection{Measure of severity—differentiating between mild and major $V C I$}

In VICCCS-1 round 4, participants considered the cognitive domains that needed assessment to measure the severity of VCI. These were reviewed in VICCCS-2 round 3, in which $94 \%$ agreed that the core domains for assessment should be executive function, attention, memory, language, and visuospatial function. The domains of learning, neuropsychiatry, and social cognition should be treated as optional, outside of the core assessment, unless and until there is stronger evidence for their inclusion. No other domains (including abstraction, agnosia, emotionality, praxis, and processing or psychomotor speed) were supported as core domains; for some, respondents thought there were insufficient tools for assessment (e.g., apraxia that features in NINDS-CSN: $80 \%$ of respondents).

Eighty-one percent of VICCCS-1 respondents felt that the differentiation between mild and major VCI (VaD) should be based on the number of domains affected and that both instrumental activities of daily living (IADLs) and activities of daily living (ADLs) were necessary determinants. In VICCCS-2 round 3, a consensus (85\%) definition was achieved for mild VCI: impairment in at least one cognitive domain, and mild to no impairment in IADL/ $A D L$, respectively (independent of the motor/sensory sequelae of the vascular event). In rounds 3 and 4 , several definitions were considered for major VCI (VaD), but no overall majority was achieved for any one option. On further examination of the various choices, $71 \%$ had chosen a definition that included the word "severe" and 73\% had chosen an option with "at least one cognitive domain" (rather than "at least two cognitive domains"). These observations were presented to the participants in round 5, 


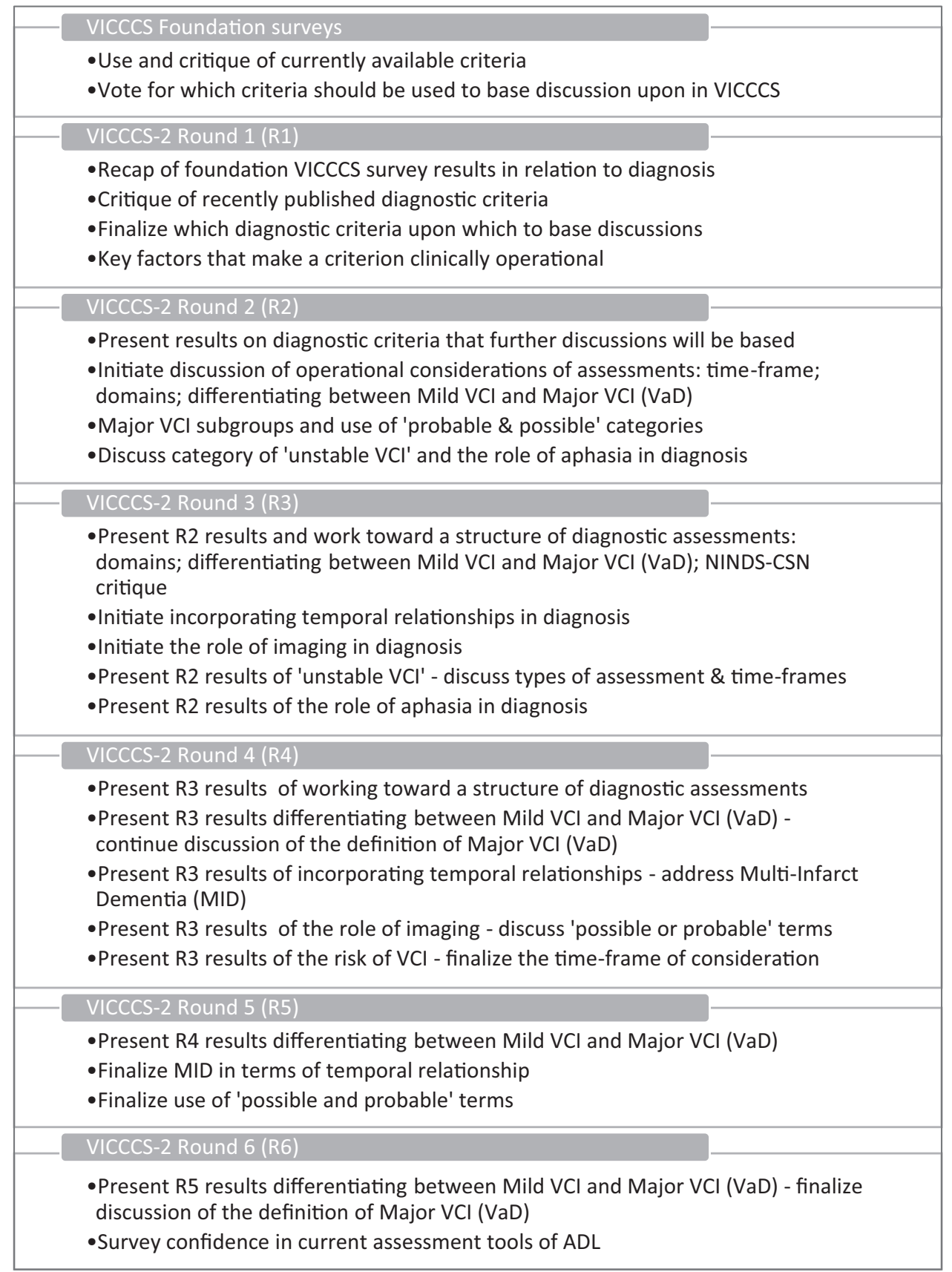

Fig. 2. Summary of topics addressed in each Delphi survey round. Abbreviations: DLB, dementia with Lewy bodies; NINDS-CSN, National Institute of Neurological Disorders-Canadian Stroke Network; PSD, post-stroke dementia; VaD, vascular dementia; VCI, vascular cognitive impairment; VICCCS, Vascular Impairment of Cognition Classification Consensus Study.

and they were asked to choose between the three mostfavored definitions from the previous round. There was $60 \%$ support for the major VCI (VaD) definition: severe deficits in at least one cognitive domain (other deficits may be present in multiple domains) and severe disruption to IADL/ADL (independent of the motor/sensory sequelae of the vascular event). Consensus-level (67\%) support was obtained for a definition requiring deficits in one rather than two cognitive domains and for inclusion of the descriptor "severe". Those who did not support the mandatory inclusion of the descriptor "severe" highlighted the important issue of people with moderate cognitive impairment and suggested the use of the term "significant" to allow greater clinical discretion and flexibility. The scenario of " $\ldots$ the case of the very bright patient whose functioning is severely compromised but still does ok on rudimentary cognitive assessment" was given as an illustrative example. 
On feedback of the results of round 5, participants were asked to consider an amendment of the definition of major VCI (VaD) by substitution of "severe" with "significant", that is, clinically significant deficits in at least one cognitive domain (other deficits may be present in multiple domains) and severe disruption to IADL/ADL (independent of the motor/sensory sequelae of the vascular event). Fifty-two percent of participants were in favor, and support for the most popular definition in round 5-severe deficits in at least one cognitive domain (other deficits may be present in multiple domains) and severe disruption to IADL/ADL (independent of the motor/sensory sequelae of the vascular event)-had dropped to 34\%. Although our predefined consensus level (67\%) was not reached for any definition in either round, it was clear that some qualification of the severity of deficit in at least one cognitive domain was preferred. Therefore, the proposed definition (representing the majority view) for major $\mathrm{VCI}(\mathrm{VaD})$ is as follows: clinically significant deficits in at least one cognitive domain (other deficits may be present in multiple domains) and severe disruption to IADL/ADL (independent of the motor/ sensory sequelae of the vascular event).

\subsubsection{Clinical evaluation and time frame for assessment}

Recommendations of the NINDS-CSN abbreviated clinical evaluation of VCI were strongly supported by round 3 respondents $(86 \%)$. However, NINDS-CSN recommendations differ for research and clinical settings, and most round 2 respondents (77\%) thought that the priority should be to agree a core of assessments for both research and clinical use, with the option of additional assessments for local use in either a clinical or research context.

It was agreed that a neuropsychological assessment protocol for use in a typical clinical diagnostic setting, noting time pressures and patient group capabilities, should take 60 minutes at most, although optional assessments could take additional time. The inclusion of all core items in the NINDS-CSN 60-minute protocol was supported by most round 3 respondents. Only the Mini-Mental State Examination supplementary test was supported by most respondents (71\%). Other supplemental tests-Rey-Osterrieth Complex Figure, Boston Naming Test, and Digit Symbol-Coding Incidental Learning-did not achieve consensus support for inclusion. All core and supplementary items in the NINDSCSN 30-minute protocol were also supported (Table 1).

\subsubsection{The role of aphasia in diagnosis of VCI}

VICCCS-2 round 2 respondents agreed $(72 \%)$ with the AHA/ASA statement on aphasia: "Severity of aphasia precludes proper cognitive assessment. However, patients with documented evidence of normal cognitive function (e.g., annual cognitive evaluations) before the clinical event that caused aphasia could be classified as having probable $\mathrm{VaD} /$ VaMCI." [22]. In round 4, respondents agreed (96\%) on the qualifying sentence "that the assessment of IADL/ADL should be made where possible". Sixty-eight percent of round 4 participants felt that "probable mild VCI or probable major VCI" was the appropriate classification of cases with aphasia when imaging was available and "possible" used in cases of aphasia when imaging was not available (90\%).

\subsubsection{Those at risk of VCI}

One of the agreed principles in VICCCS-1 [25] was as follows: "The new VCI construct recognizes the importance of people who are at risk of VCI, however, their consideration should be contingent upon the presentation of a sustained level of impairment even if in a very mild form as opposed to impairment that can be transient or revert to normal levels". Respondents agreed (96\%) that people at risk of VCI should be given greater consideration for diagnosis if at least 6 months of sustained impairment is present. Eighty-eight percent also agreed that in those at risk of VCI, other potential causes of sustained impairment (e.g., depression or vitamin D deficiency), in addition to the already agreed exclusions from diagnosis (i.e., drug/ alcohol abuse/dependence within the last 3 months of first

Table 1

NINDS-CSN Neuropsychological assessment supported for use in VICCCS guidelines

\begin{tabular}{lrr}
\hline Assessment tool & 60 minute & 30 minute \\
\hline Animal naming (semantic fluency) & $100 \%$ & $100 \%$ \\
Controlled Oral Word Association (phonemic fluency) & $86 \%$ & $83 \%$ \\
WAIS-III Digit Symbol-Coding (processing speed and activation) & $85 \%$ & $82 \%$ \\
Trail Making Test (processing speed and set shifting) & $95 \%$ & $75 \%$ (Supplementary) \\
Revised Hopkins Verbal Learning Test (additional scoring options: strategic & & $79 \%$ \\
$\quad$ learning; episodic memory; and executive organization) & $80 \%$ & $93 \%$ \\
Rey-Osterrieth Complex Figure Copy (visuospatial) & $96 \%$ & $86 \%$ \\
Boston Naming Test, 2nd Edition, Short Form (visual confrontation naming) & $71 \%$ & $71 \%$ \\
Simple and choice reaction time tasks & $71 \%$ & $75 \%$ \\
Neuropsychiatric Inventory, Questionnaire Version (NPI-Q) & & \\
Center for Epidemiological Studies-Depression Scale (CES-D), Short Form & & \\
Mini-Mental State Examination (MMSE; Supplementary Material) & & \\
\hline
\end{tabular}

Supported tests from the proposed NINDS-CSN [13].

NOTE. 30- or 60-minute protocols are listed with percentage support, where applicable. Similar word list learning tests to the revised Hopkins verbal learning test would be necessary for non-English speakers. 
recognition of impairment or delirium), should have been excluded. Caregiver reporting (88\%) and clinical observation $(73 \%)$ were supported mechanisms to collect this information. Screening-type assessment (49\%) and more detailed formal assessment (43\%) were not supported by the majority.

\subsubsection{Possible and probable terms in VCI and "mixed dementias" subgroup}

\subsubsection{Top of form}

In the AHA/ASA, which served as a starting point to discuss the use of the terms "possible" and "probable" VCI, only the categories of "possible" VaD or vascular mild cognitive impairment (VaMCI) allowed the inclusion of other phenotypes (e.g., evidence of other neurodegenerative disorders). The AHA/ ASA also does not provide separate diagnosis or allow delineation of further subgroups of patients (e.g., the comorbidities present in mixed dementias). In VICCCS-1, the consensus was that VCI should contain a standalone umbrella-like subgroup termed "mixed dementias". VICCCS-1 participants also agreed that this subgroup could comprise further groupings that included patients with specific combinations of phenotypes, each of which would be specifically named where possible (e.g., VCI-Alzheimer's disease (AD) if there is evidence of both VCI and AD). Furthermore, it was agreed that the order of the descriptive phenotypic terms relevant to patients should attempt to reflect the relative contribution of phenotypes present, that is, $\mathrm{AD}-\mathrm{VCI}$, or VCI-AD, to the extent that discrimination was possible.

On consideration of differences between the AHA/ASA and VICCCS-1, VICCCS-2 round 2 respondents agreed (92\%) that the diagnostic guidelines should attempt to incorporate the VICCCS-proposed "mixed dementias" as a separate distinct diagnostic subgroup of major VCI, and "probable" or "possible" are to be used to differentiate the level of diagnostic evidence to help classify patients for both major VCI (VaD) subgroups (81\%) and mild VCI (70\%).

\subsubsection{Incorporating temporal relationships in VCI diagnosis}

In AHA/ASA, a clear temporal relationship between a vascular event and onset of cognitive deficits is required for a "probable" diagnosis. In VICCCS-1, the definition of PSD — an agreed subgroup of major VCI (VaD)—required that cognitive decline develop within 6 months of stroke. However, temporal relationships were not discussed for other subtypes of VCI. In VICCCS-2, respondents thought that a clear temporal relationship between a vascular event and onset of cognitive deficits should not be an essential component for diagnosis of mild VCI (77\%), SIVaD (88\%), mixed dementias (85\%), or MID (74\%).

\subsubsection{The role of imaging in VCI diagnosis}

In VICCCS-2 round 3, the consensus was that imaging evidence of CVD was essential for diagnosis of major
VCI (VaD) (86\%) and mild VCI (79\%). In round 4, although most respondents felt the NINDS-CSN recommendations were possible/appropriate in the respondents' clinical settings $(75 \%$ magnetic resonance imaging [MRI] $/ 81 \%$ computed tomography [CT]), 93\% thought that the "acceptable MRI measures" proposed in NINDSCSN should be the core recommendation for imaging in clinical settings, and the "recommended MRI measures" supported as additional measures for use in either clinical or research contexts (Table 2 and 3). Several respondents stated that $\mathrm{CT}$ might be insensitive or insufficient to detect small-vessel disease or evaluate vascular status. The limitations of CT for VCI are also highlighted in NINDS-CSN. In round 4, respondents agreed (93\%) that MRI should be the gold-standard imaging for VCI, and 90\% agreed that CT should be used only if MRI were not available or deemed too costly. This would also apply to cases where MRI is contraindicated. A consensus (68\%) was reached on the use of the term "possible" mild VCI or major VCI if neither MRI nor CT imaging were available. Yet $89 \%$ felt that "probable" was the appropriate diagnostic category if only CT imaging were available.

Majority of support (67\%) was not reached for any of the other "future" imaging methods described in NINDS-CSN, and the consensus was that none of these is ready for inclusion in clinical diagnosis.

A summary of VICCCS diagnosis guidelines is provided in Box 1.

\section{Discussion}

The VICCCS-2 has provided new consensus-based guidelines for diagnosis of mild and major VCI ( $\mathrm{VaD})$ as previously defined [25], with AHA/ASA and NINDSCSN guidelines as reference points for discussions. Clinical evaluation and neuropsychological protocols in the NINDS-CSN were supported for use by VICCCS respondents. The "acceptable MRI measures" outlined in NINDS-CSN should be the core recommendation for imaging in clinical settings. In terms of assessing severity, VICCCS diagnosis guidelines specify deficits in at least 1 domain, with clinically significant cognitive deficits of sufficient severity (moderate to severe) and severe deficits in IADLs/ADLs differentiating major from mild forms. Patients with PSD, SIVaD, MID, and mixed dementias should also be subcategorized and any comorbid neurodegenerative disease recorded.

\subsection{Comparison of VICCCS with recently published guidelines}

The VICCCS was conducted between 2010 and 2013, which coincided with the development of DSM-5 and VASCOG criteria for VCDs [23]. VICCCS participants provided collective feedback on draft DSM-5 proposals 
Table 2

MRI and CT imaging recommendations adapted from NINDS-CSN recommendations

\begin{tabular}{|c|c|c|c|}
\hline Feature & Core MRI measures & Additional MRI measures & CT measures \\
\hline WMHs & $\begin{array}{l}\text { - Preferred: ARWMC scale [35] } \\
\text { - Acceptable: CHS WMH Scale [32] }\end{array}$ & $\begin{array}{l}\text { - Quantitative measurement of WMH } \\
\text { volume normalized for head size } \\
\text { - Anatomical mapping also } \\
\text { encouraged [32] }\end{array}$ & $\begin{array}{l}\text { - Diffuse white matter: ARWMC } \\
\text { scale [35] }\end{array}$ \\
\hline Infarction & $\begin{array}{l}\text { - Number and size at specified locations: } \\
\text { - Size (largest diameter): large }> \\
1.0 \mathrm{~cm} \text {, small = }=3-10 \mathrm{~mm} \\
\text { - Location (encourage use of Talairach } \\
\text { Atlas [36] for precision) } \\
\circ \text { Anatomical locations: } \\
\text { - Supratentorial } \\
\text { - Hemisphere } \\
\text { - Cortical (may include } \\
\text { subcortical) } \\
\text { - Exclusively subcortical white } \\
\text { matter } \\
\text { - Exclusively subcortical gray } \\
\text { matter } \\
\text { Infratentorial }\end{array}$ & $\begin{array}{l}\text { - All infarcts localized using a standard } \\
\text { approach to generate quantitative } \\
\text { measures of volume and location. } \\
\text { Ideally, identified infarcts would also } \\
\text { be mapped to a common stereotactic } \\
\text { space [32] } \\
\text { - All infarcts should be differentiated } \\
\text { from perivascular spaces (Virchow- } \\
\text { Robin spaces) by CHS criteria (see } \\
\text { Table 3), independently }\end{array}$ & $\begin{array}{l}\text { - Discrete hypodensities } \\
\text { - Cerebrospinal fluid density consistent } \\
\text { with infarction or old hemorrhage: } \\
\text { - Small }>3-1.0 \mathrm{~mm} \\
\text { - Large }>1.0 \mathrm{~mm} \\
\text { - Acute hemorrhage } \\
\text { - Number, volume, and location-as } \\
\text { core MRI }\end{array}$ \\
\hline Hemorrhage & $\begin{array}{l}\text { - Number and size in each location } \\
\text { - Size (largest diameter) } \\
\text { - Large }>1 \mathrm{~cm} \text { in diameter } \\
\text { - Microhemorrhage }<1 \mathrm{~cm} \\
\text { susceptibility on gradient echo } \\
\text { - Must report lower size limit cutoff, } \\
\text { field strength } \\
\text { - Location-as infarcts }\end{array}$ & $\begin{array}{l}\text { All lesions localized using a standard } \\
\text { approach to generate quantitative } \\
\text { measures of volume and location. } \\
\text { Ideally, identified lesions would also be } \\
\text { mapped to a common stereotactic } \\
\text { space [32] }\end{array}$ & \\
\hline
\end{tabular}

Abbreviations: ARWMC, age-related white matter change; CHS, Cardiovascular Health Study; AVMs, arteriovenous malformations; CT, computed tomography; MRI, magnetic resonance imaging; WMH, white matter hyperintensity.

that were made available before their finalization, through a tailored survey developed (by O.A.S), in consultation with P.S. acting on behalf of the DSM-5 Neurocognitive Disorders Work Group. VICCCS respondents agreed that the mild and major terminologies proposed in the DSM-5 were helpful and should be adopted in VICCCS. The VASCOG criteria are also aligned with the DSM-5 [23].
In terms of assessing severity, both AHA/ASA and VICCCS specify the same core domains for assessment. In VASCOG, visuoconstructional-perceptual ability, praxisgnosis-body schema, and social cognition are additionally tested. The definitions of mild VCI are comparable; however, they are not subcategorized in VICCCS as respondents felt more evidence was needed. The definition of major forms of VCI in VICCCS and VASCOG requires deficits

Table 3

Signal characteristics that differentiate perivascular space and infarcts

\begin{tabular}{llll}
\hline Measurable feature & $\mathrm{T} 1$ & FLAIR/Proton Density & T2 \\
\hline Perivascular space & Decreased & Isointense & Increased \\
Ischemic change & Isointense (N/A in gray matter) & Increased (N/A in gray matter) & Increased (N/A in gray matter) \\
Infarct & Decreased (decreased or isointense in gray matter) & Increased & Increased \\
\hline
\end{tabular}

Adapted from NINDS-CSN [13].

NOTE. Differences seen in gray matter from white matter are noted. 
Box 1

Summary of the Vascular Impairment of Cognition Classification Consensus Study (VICCCS) diagnosis guidelines

Definitions and diagnosis of VCI:

Clinical evaluation and neuropsychological protocols as provided in the National Institute of Neurological DisordersCanadian Stroke Network guidelines are supported (Table 1). Core domains for assessment should include executive function, attention and memory, as well as language and visuospatial function.

Mild VCI: Impairment in at least one cognitive domain and mild to no impairment in instrumental activities of daily living (IADLs)/activities of daily living (ADLs), respectively (independent of the motor/sensory sequelae of the vascular event).

Major VCI (VaD): Clinically significant deficits of sufficient severity in at least one cognitive domain (deficits may be present in multiple domains) and severe disruption to IADLs/ADLs (independent of the motor/sensory sequelae of the vascular event).

Patients given a diagnosis of major VCI $(\mathrm{VaD})$ are subcategorized according to the underlying pathology as appropriate (Fig. 1). A clear temporal relationship (within 6 months) between a vascular event and onset of cognitive deficits is only required for a diagnosis of post-stroke dementia (PSD).

Subtypes of major VCI $(\mathrm{VaD}) \dagger$ :

Post-stroke dementia:

A patient described as having PSD may or may not have presented evidence of mild cognitive impairment before stroke. The patient will exhibit immediate and/or delayed cognitive decline that begins within 6 months after a stroke and that does not reverse. PSD can result from several different vascular causes and changes in the brain. It encompasses dementia that develops within 6 months of stroke in patients with multiple cortical-subcortical infarcts and strategic infarcts; patients with subcortical ischemic vascular dementia; and those with various forms of neurodegenerative pathology, including Alzheimer's disease (AD). The temporal relationship between the cognitive decline and the stroke differentiates PSD from other forms of major VCI (VaD).

Mixed dementias:

A standalone umbrella subgroup termed "mixed dementias" includes phenotypes representing each combination between vascular and neurodegenerative disease, that is, VCI-AD, VCI-dementia with Lewy bodies, and so forth. It is recommended that a patient is referred to as having "VCI-AD", for example, according to the clinically probable phenotypes, rather than the less-specific "mixed dementia". Where discrimination is possible, the order of terms should reflect the probable relative contribution of the underlying pathology, that is, AD-VCI or VCI-AD.

Subcortical ischemic vascular dementia:

Small-vessel disease is the main vascular cause of subcortical ischemic vascular dementia. Lacunar infarcts and ischemic white matter lesions are the main type of brain lesions, which are located predominantly subcortically. This diagnosis incorporates the overlapping clinical entities of Binswanger's disease and the lacunar state.

Multi-infarct dementia:

Multi-infarct dementia is used to indicate the presence of multiple large cortical infarcts and their likely contribution to the dementia.

"Probable" and "possible" - terms for the availability of evidence:

Magnetic resonance imaging is a "gold-standard" requirement for a clinical diagnosis of VCI. Probable mild VCI or probable major $\mathrm{VCI}(\mathrm{VaD})$ is the appropriate diagnostic category if computed tomography imaging is the only means of imaging available. Recommendations on imaging from the National Institute of Neurological Disorders-Canadian Stroke Network should be followed (Table $2 \&$ 3). Possible mild VCI or possible major VCI (VaD) would be appropriate diagnoses if neither MRI nor computed tomography imaging were available.

In diagnosis of VCI when full clinical assessment of the cognitive impairment resulting from the clinical event is impaired by aphasia, patients with documented evidence of normal cognitive function (e.g., annual cognitive evaluations) before the clinical event that caused aphasia could be classified as having probable mild VCI or major VCI (VaD) if imaging is available, and the assessment of ADLs should be made where possible. If imaging is not available, the classification should be possible mild VCI or major VCI (VaD).

Those at risk of VCI:

It is recommended that greater consideration for diagnosis be given to people who are at risk of VCI if they present with at least 6 months of sustained impairment (even if very mild), rather than transient impairment, as identified through caregiver reporting and clinical observation. All other potential causes of sustained impairment (e.g., depression or vitamin D deficiency, in addition to the already agreed exclusions from diagnosis) should have been excluded.

Exclusions from diagnosis:

Drug/alcohol abuse/dependence within the last 3 months of first recognition of impairment or delirium.

*Clinically significant deficits include moderate severity. Cognitive impairment in mild VCI is differentiated from major (VaD) by not being clinically significant. $\dagger$ Definitions were agreed in VICCCS-1 and supported in VICCCS-2. 
in at least 1 domain, rather than 2 domains as specified in AHA/ASA. Loss of independence in IADLs is the threshold in VASCOG and DSM-5. In VICCCS-1, assessment of both IADLs and ADLs was deemed necessary to determine severity. Patients with PSD, SIVaD, and MID are also subcategorized in VICCCS but not specified in AHA/ASA, although subtypes in VASCOG include cortical-subcortical and subcortical ischemic. The AHA/ASA category of "unstable VaMCI", for cases that revert to normal from VaMCI, was not supported by VICCCS respondents.

One of the main differences between VICCCS and AHA/ ASA is the VICCCS subcategorization of patients with comorbid pathologies, seen to be important, under the umbrella term "mixed dementias" but with the types of pathologies specified. Improvements in the accuracy of this would be an important goal of any future operational diagnostic protocols, for which research into biomarkers may be helpful [37]. The AHA/ASA does not delineate these subgroups of patients, with only the categories of "possible" VaD or VaMCI allowing the inclusion of other phenotypes (e.g., evidence of other neurodegenerative disorders). VASCOG criteria encompass categories of multiple causation, including VCD with concomitant AD (major or mild) and VCD with additional pathology: for example, Lewy body disease.

In VICCCS, the use of probable and possible terms is reserved for the strength of evidence to support diagnosis. One such example of supportive evidence is a clear temporal relationship between a vascular event and onset of cognitive deficits in PSD, which is differentiated from other forms of VCI by the onset of symptoms within 6 months of stroke. Another is the imaging of CVD. VICCCS respondents concluded that MRI is the gold standard for imaging. If CT were the only means available, only a "probable" diagnosis could be made, and only a diagnosis of "possible" if no imaging evidence were available. The recent STRIVE imaging criteria for small-vessel disease [38] published after the completion of our study, aligned with VICCCS' conceptualization for SIVaD, warrant consideration in future validations of VICCCS-2. VICCCS supports and expands on the AHA/ASA guidance on patients with aphasia, in whom assessment of IADLs/ADLs should be made when possible, allowing diagnosis of "probable" with imaging evidence or "possible" without it.

\subsection{Limitations and future work}

The use of online Delphi surveys in VICCCS allowed flexible and confidential participation among an unprecedented number of international participants over an extended period $[25,26,39]$. The guidelines reflect considered opinion because there was little participant attrition between rounds (90\%-97\% of participants responding over rounds 2-6), reflecting sustained engagement, with most topics addressed over multiple rounds.
Given the large number of respondents and their broad interdisciplinarity, it is noteworthy that we achieved consensus on most topics addressed in VICCCS. Reaching consensus was most challenging for the definition of major VCI (VaD), perhaps reflecting residual sensitivities associated with this diagnosis and associated implications for health and social care services. Consequently, specific thresholds of severity of impairment were not established. VICCCS-1 concluded that subtyping of mild VCI may be worthwhile but required more research [25], a view supported in a recent study that highlighted the importance of harmonizing neuropsychological test score levels for defining impairment [40]. Inclusion of defined thresholds of severity of impairment within the neuropsychological test battery and IADLs/ ADLs may help to guide the differentiation of VCI subtypes in clinical settings. Support for the continued use of MiniMental State Examination [41] may be surprising, given that MoCA [42] has been shown to be an equivalent or more sensitive test for the detection of VCI, $([43,44]$, for example). It is noteworthy that only $23 \%$ of respondents indicated use of the NINDS-CSN 5-minute protocol, (see Supplementary Material), while NINDS-CSN endorsed the full and the short MoCA. The use of biomarkers and advances in imaging criteria $[38,45,46]$, including the use of arterial spin-labeling MRI [47,48], may refine the subtyping of mild and major VCI. Further work is needed on imaging protocols, including measurement of gray matter atrophy, cortical thinning or global atrophy in the context of suspected VCI $[49,50]$. Given the presence of vascular pathologies in apparent cognitively normal elderly people, recent progress in establishing neuropathological diagnostic criteria for assessing the likelihood that CVD contributed to premortem cognitive impairment [51] is likely to have an important role in the validation of future premortem diagnostic approaches. Translational models and genetic studies may provide further insight into the pathological mechanisms and possible therapeutic targets for VCI [52]. A multimodal strategy for treatment of VCI, incorporating both nonpharmacological therapies, such as transcranial magnetic stimulation ([53], for review), and pharmacological treatment, has been proposed [54].

\section{Conclusions}

We present a consensus-based set of guidelines for diagnosing VCI, supported by a large multinational group of researchers. VICCCS guidelines have drawn upon, critiqued, expanded, and refined previous efforts. We hope that they will be widely adopted, to improve consistency in diagnosis and standardization in VCI research. This would allow better comparison of findings across studies and facilitate largescale collaborative research on a group of diseases that despite modest prevalence and considerable heterogeneity have major societal impact. 


\section{Acknowledgments}

O.A.S. was the study coordinator, analyzed the data, formulated the questionnaires, and wrote the manuscript. P.G.K. was the chief investigator, conceived and designed the study, obtained the necessary funding, reviewed each round data, formulated the questionnaires, and wrote the manuscript. Y.B.-S., A.P.P., and S.L. were co-investigators and members of the Steering Group. Other listed authors were members of the Steering Group, who reviewed the content of the pilot questionnaires, draft and final manuscript, and were participants in the study. Authors listed under the banner of VICCCS groups contributed to data gathering in multiple survey rounds and approved the final submitted version of the article.

VICCCS group-Argentina: F. E. Taragano, CONICET National Research Council and CEMIC University Hospital; Australia: J. Kril, University of Sydney; Austria: M. Cavalieri, Medical University of Graz; K. A. Jellinger, Institute of Clinical Neurobiology; and G. G. Kovacs, Medical University of Vienna; Belgium: S. Engelborghs, University of Antwerp and C. Lafosse, RevArte Rehabilitation Hospital and Catholic University of Leuven; Brazil: P. H. Bertolucci, Universidade Federal de Sao Paulo; S. Brucki, University of Sao Paulo; P. Caramelli, Universidade Federal de Minas Gerais; and T .C. de Toledo Ferraz Alves, Department of Psychiatry of São Paulo Medical School; Canada: C. Bocti, Université de Sherbrooke; T. Fulop, Université de Sherbrooke; D. B. Hogan, University of Calgary; G. R. Hsiung, University of British Columbia; A. Kirk, University of Saskatchewan; L. Leach, Glendon College, York University; A. Robillard, Hopital Maisonneuve; and D. J. Sahlas, McMaster University. People's Republic of China: Q. Guo, Huashan Hospital, Fudan University and J. Tian, Dongzhimen Hospital, Beijing University of Chinese Medicine; Finland: L. Hokkanen, University of Helsinki and H. Jokinen, Helsinki University Hospital; France: S. Benisty, Institution Nationale des Invalides; V. Deramecourt, Lille University Hospital; J. Hauw, APHP, Pitié-Salpêtrière Hospital, and Pierre et Marie-Curie University; and H. Lenoir, Broca HospitalHUPC-APHP and Paris-Descartes 5 University; Greece: M. Tsatali, Greek Alzheimer Association and M. Tsolaki, Aristotle University of Thessaloniki; India: U. Sundar, Lokmanya Tilak Municipal Medical College and Hospital, Sion, Mumbai; Ireland: R. F. Coen, Mercer's Institute for Research on Ageing, St. James's Hospital, Dublin; Israel: A. D. Korczyn, Tel Aviv University; Italy: M. Altieri, Sapienza Università di Roma; M. Baldereschi, Italian National Research Council; C. Caltagirone, Rome University of Tor Vergata and Santa Lucia IRCCS Foundation Rome; G. Caravaglios, Azienda Ospedaliera Cannizzaro, Catania; A. Di Carlo, Institute of Neuroscience, Italian National Research Council; V. Di Piero, Sapienza University; G. Gainotti, Catholic University; S. Galluzzi, IRCCS Istituto Centro San
Giovanni di Dio-Fatebenefratelli; G. Logroscino, University of Bari; P. Mecocci, University of Perugia; D. V. Moretti, IRCCS Istituto Centro San Giovanni di DioFatebenefratelli; and A. Padovani, Università degli Studi di Brescia; Japan: T. Fukui, Kawasaki Memorial Hospital; M. Ihara, Kyoto University and T. Mizuno, Kyoto Prefectural University of Medicine; Republic of Korea: S. Y. Kim, Seoul National University Bundang Hospital; Nigeria: R. Akinyemi, University of Ibadan and Newcastle University, UK; O. Baiyewu, University of Ibadan; and A. Ogunniyi, University of Ibadan; Poland: A. Szczudlik, Jagiellonian University Medical College; Portugal: A. J. Bastos-Leite, University of Porto; H. Firmino, Coimbra University Hospital; J. Massano, University of Porto and Hospital Pedro Hispano/ULS Matosinhos; and A. Verdelho, University of Lisbon, Hospital de Santa Maria; Russia: L. S. Kruglov, St. Petersburg University and St. Petersburg Psychoneurological Research Institute; Singapore: M. K. Ikram, National University of Singapore, Erasmus Medical Centre, Rotterdam and N. Kandiah, National Neuroscience Institute; Spain: E. Arana, Fundación IVO; J. Barroso-Ribal, University of La Laguna; T. Calatayud, Hospital Universitario Central de Asturias; A. J. Cruz-Jentoft, Hospital Universitario Ramón y Cajal Madrid; S. López-Pousa, Institut Català de la Salut, Girona and Institut d'Assistència Sanitària, Catalonia; P. Martinez-Lage, Fundacion CITA Alzheimer; and M. Mataro, University of Barcelona; Sweden: A. Börjesson-Hanson, Neuroscience and Physiology, Sahlgrenska Academy at the University of Gothenburg; E. Englund, Lund University; E. J. Laukka, Karolinska Institutet; C. Qiu, Karolinska Institutet; and M. Viitanen, Karolinska Institutet, University of Turku; The Netherlands: G. J. Biessels, University Medical Center Utrecht; F.-E. de Leeuw, Radboud University Nijmegen Medical Centre and Donders Institute Brain Cognition \& Behaviour; T. den Heijer, Sint Franciscus Gasthuis; L. G. Exalto, UMCU; L. J. Kappelle, University Medical Centre Utrecht; N. D. Prins, VU University Amsterdam; E. Richard, University of Amsterdam and Radboud University, Nijmegen; B. Schmand, University of Amsterdam; E. van den Berg, University Medical Center Utrecht; and W. M. van der Flier, VU University Medical Center; Turkey: B. Bilgic, Istanbul University; United Kingdom: L. M. Allan, Newcastle University; J. Archer, MidYorkshire NHS Trust; J. Attems, Newcastle University; A. Bayer, Cardiff University; D. Blackburn, University of Sheffield; C. Brayne, Cambridge Institute of Public Health, University of Cambridge; R. Bullock, Kingshill Research Centre; P. J. Connelly, University of Dundee, Murray Royal Hospital, Perth; A. Farrant, NHS; M. Fish, Musgrove Park Hospital; K. Harkness, Sheffield Teaching Hospital Foundation Trust; P. G. Ince, University of Sheffield; P. Langhorne, Glasgow University; J. Mann, The Research Institute for the Care of Older People; F. E. Matthews, MRC Biostatistics Unit; P. Mayer, 
Institute of Ageing West Midlands; S. T. Pendlebury, Centre for Prevention of Stroke and Dementia, University of Oxford and NIHR Oxford Biomedical Research Centre; R. Perneczky, Imperial College London; R. Peters, Imperial; D. Smithard, King's College Hospital, London and University of Kent; B. C. Stephan, Newcastle University; J. E. Swartz, Bracket Global; S. Todd, Western Health and Social Care Trust; D. J. Werring, Stroke Research Centre, UCL Institute of Neurology; S. N. Wijayasiri, Bedford Hospital; G. Wilcock, University of Oxford; G. Zamboni, Nuffield Department of Clinical Neurosciences (NDCN), University of Oxford; and United States of America: R. $\mathrm{Au}$, Boston University; S. Borson, University of Washington School of Medicine; A. Bozoki, Michigan State University; J. N. Browndyke, Duke University Medical Center; M. M. Corrada, University of California, Irvine; P. K. Crane, University of Washington; B. S. Diniz, University of Texas Health Science Center at Houston; L. Etcher, Spring Arbor University; H. Fillit, The Alzheimer's Drug Discovery Foundation; S. M. Greenberg, Massachusetts General Hospital and Harvard Medical School; L. T. Grinberg, University of California San Francisco and University of Sao Paulo Medical School; S. W. Hurt, Weill Cornell Medical College; M. Lamar, University of Illinois at Chicago and Institute of Psychiatry, King's College London, UK; M. Mielke, Mayo Clinic; B. R. Ott, Brown University; G. Perry, University of Texas at San Antonio; W. J. Powers, University of North Carolina; C. Ramos-Estebanez, Case Western Reserve University; B. Reed, University of California, Davis; R. O. Roberts, Mayo Clinic; J. R. Romero, Boston University; A. J. Saykin, Indiana University; S. Seshadri, Boston University; L. Silbert, Oregon Health \& Science University; Y. Stern, Columbia University; C. Zarow, University of Southern California.

This work was supported by a project grant (Ref117) from the Alzheimer's Society (UK).

G.A.F. reports personal fees from Pfizer, Athersys, AstraZeneca, Lundbeck, Cerevast, and Daiichi Sankyo, and grants and personal fees from Boehringer Ingelheim, outside the submitted work. J. O'B. reports personal fees from GE Healthcare, TauRx, and Cytox, and grants and personal fees from Avid/Lilly, outside the submitted work. I.S. reports personal fees and other from Takeda, outside the submitted work. Outside the submitted work, S.E.B. reports institutional grants from Pfizer, GE Healthcare, Eli Lilly, Elan/Transition Therapeutics, Roche, and Cognoptix, and personal fees from Pfizer, GE Healthcare, Eli Lilly, Eisai, Boehringer Ingelheim, and Novartis.

\section{Supplementary data}

Supplementary data related to this article can be found at https://doi.org/10.1016/j.jalz.2017.09.007.

\section{RESEARCH IN CONTEXT}

1. Systematic review: Criteria for diagnosis of vascular cognitive impairment (VCI) were reviewed and refined through several rounds of a multinational Delphi survey, after PubMed search identified 15 articles (published before August 2010) proposing diverse diagnostic criteria for diagnosis of multiinfarct dementia; vascular dementia (VaD), subcortical (ischemic) VaD, VCI, and vascular cognitive disorders. The manuscript author lists identified most of the study participants, and a Steering Group.

2. Interpretation: This survey yielded consensus-based guidelines for the diagnosis of VCI that have drawn upon and refined previous proposals. The high levels of participation by the research community demonstrate endorsement, and should promote widespread adoption of the guidelines.

3. Future directions: Consensus research priorities include: evidence-based studies to (i) determine appropriate subdivision of mild VCI, and define thresholds for severity of impairment; (ii) improve phenotyping of concurrent pathology in mixed dementias or psychiatric disorders that present with cerebrovascular disease.

\section{References}

[1] Hachinski VC, Lassen NA, Marshall J. Multi-infarct dementia. A cause of mental deterioration in the elderly. Lancet 1974;2:207-10.

[2] Kalaria RN, Kenny RA, Ballard CG, Perry R, Ince P, Polvikoski T. Towards defining the neuropathological substrates of vascular dementia. J Neurol Sci 2004;226:75-80.

[3] Sachdev P. Vascular cognitive disorder. Int J Geriatr Psychiatry 1999; 14:402-3.

[4] Rockwood K, Macknight C, Wentzel C, Black S, Bouchard R, Gauthier S, et al. The diagnosis of "mixed" dementia in the Consortium for the Investigation of Vascular Impairment of Cognition (CIVIC). Ann N Y Acad Sci 2000;903:522-8.

[5] O'Brien JT, Erkinjuntti T, Reisberg B, Roman G, Sawada T, Pantoni L, et al. Vascular cognitive impairment. Lancet Neurol 2003;2:89-98.

[6] Zhao QL, Zhou Y, Wang YL, Dong KH, Wang YJ. A new diagnostic algorithm for vascular cognitive impairment: the proposed criteria and evaluation of its reliability and validity. Chin Med J (Engl) 2010; 123:311-9.

[7] Cao X, Guo Q, Zhao Q, Jin L, Fu J, Hong Z. The neuropsychological characteristics and regional cerebral blood flow of vascular cognitive impairment-no dementia. Int J Geriatr Psychiatry 2010; 25:1168-76.

[8] Hachinski VC, Bowler JV. Vascular dementia. Neurology 1993; 43:2159-60; author reply 2160-1.

[9] Roman GC, Sachdev P, Royall DR, Bullock RA, Orgogozo JM, LopezPousa S, et al. Vascular cognitive disorder: a new diagnostic category updating vascular cognitive impairment and vascular dementia. J Neurol Sci 2004;226:81-7. 
[10] Rockwood K, Wentzel C, Hachinski V, Hogan DB, MacKnight C, McDowell I. Prevalence and outcomes of vascular cognitive impairment. Vascular Cognitive Impairment Investigators of the Canadian Study of Health and Aging. Neurology 2000;54:447-51.

[11] Chui HC, Victoroff JI, Margolin D, Jagust W, Shankle R, Katzman R. Criteria for the diagnosis of ischemic vascular dementia proposed by the State of California Alzheimer's Disease Diagnostic and Treatment Centers. Neurology 1992;42:473-80.

[12] Erkinjuntti T, Inzitari D, Pantoni L, Wallin A, Scheltens P, Rockwood K, et al. Research criteria for subcortical vascular dementia in clinical trials. J Neural Transm Suppl 2000;59:23-30.

[13] Hachinski V, Iadecola C, Petersen RC, Breteler MM, Nyenhuis DL, Black SE, et al. National Institute of Neurological Disorders and Stroke-Canadian Stroke Network vascular cognitive impairment harmonization standards. Stroke 2006;37:2220-41.

[14] Hachinski VC, Iliff LD, Zilhka E, Du Boulay GH, McAllister VL, Marshall J, et al. Cerebral blood flow in dementia. Arch Neurol $1975 ; 32: 632-7$.

[15] World Health Organization. Diagnostic criteria for research. The ICD10 classification of mental and behavioural disorders. Geneva: World Health Organization; 1993.

[16] Roman GC, Tatemichi TK, Erkinjuntti T, Cummings JL, Masdeu JC, Garcia JH, et al. Vascular dementia: diagnostic criteria for research studies. Report of the NINDS-AIREN International Workshop. Neurology 1993;43:250-60.

[17] American Psychiatric Association. Diagnostic and statistical manual of mental disorders. 4th ed. Washington, DC: American Psychiatric Association; 1994.

[18] American Psychiatric Association Diagnostic and statistical manual of mental disorders 5th ed. Arlington: American Psychiatric Publishing; 2013

[19] Chui HC, Mack W, Jackson JE, Mungas D, Reed BR, Tinklenberg J, et al. Clinical criteria for the diagnosis of vascular dementia: a multicenter study of comparability and interrater reliability. Arch Neurol 2000;57:191-6.

[20] Wiederkehr S, Simard M, Fortin C, van Reekum R. Validity of the clinical diagnostic criteria for vascular dementia: a critical review. Part J Neuropsychiatry Clin Neurosci 2008;20:162-77.

[21] Pohjasvaara T, Mantyla R, Ylikoski R, Kaste M, Erkinjuntti T. Comparison of different clinical criteria (DSM-III, ADDTC, ICD-10, NINDS-AIREN, DSM-IV) for the diagnosis of vascular dementia. National Institute of Neurological Disorders and Stroke-Association Internationale pour la Recherche et l'Enseignement en Neurosciences. Stroke 2000;31:2952-7.

[22] Gorelick PB, Scuteri A, Black SE, Decarli C, Greenberg SM, Iadecola C, C.o.E.American Heart Association Stroke CouncilC.o.C.N.C.o.C.R. Prevention, Intervention, S. Council on Cardiovascular, and Anesthesia, et al. Vascular contributions to cognitive impairment and dementia: a statement for healthcare professionals from the american heart association/american stroke association. Stroke 2011; 42:2672-713.

[23] Sachdev P, Kalaria R, O’Brien J, Skoog I, Alladi S, Black SE, et al.B. Internationlal Society for Vascular, and D. Cognitive. Diagnostic criteria for vascular cognitive disorders: a VASCOG statement. Alzheimer Dis Assoc Disord 2014;28:206-18.

[24] Hachinski V, Oveisgharan S, Romney AK, Shankle WR. Optimizing the Hachinski Ischemic Scale. Arch Neurol 2012;69:169-75.

[25] Skrobot OA, O'Brien J, Black S, Chen C, DeCarli C, Erkinjuntti T, et al. The Vascular Impairment of Cognition Classification Consensus Study. Alzheimers Dement 2017;13:624-33.

[26] Dalkey N, Helmer O. An experimental application of the Delphi method to the use of experts. Management Sci 1963;9:458-67.

[27] Alexandrov AV, Pullicino PM, Meslin EM, Norris JW. Agreement on disease-specific criteria for do-not-resuscitate orders in acute stroke. Members of the Canadian and Western New York Stroke Consortiums. Stroke 1996;27:232-7.
[28] Rosen WG, Terry RD, Fuld PA, Katzman R, Peck A. Pathological verification of ischemic score in differentiation of dementias. Ann Neurol 1980;7:486-8.

[29] Knopman DS, Rocca WA, Cha RH, Edland SD, Kokmen E. Incidence of vascular dementia in Rochester, Minn, 1985-1989. Arch Neurol 2002;59:1605-10

[30] Price CC, Jefferson AL, Merino JG, Heilman KM, Libon DJ. Subcortical vascular dementia: integrating neuropsychological and neuroradiologic data. Neurology 2005;65:376-82.

[31] Roman GC, Tatemichi TK, Erkinjuntti T, Cummings JL, Masdeu JC, Garcia JH, et al. VASCULAR dementia - diagnostic-criteria for research studies - report of the NINDS-AIREN International Workshop. Neurology 1993;43:250-60.

[32] Yue NC, Arnold AM, Longstreth WT Jr, Elster AD, Jungreis CA, O'Leary DH, et al. Sulcal, ventricular, and white matter changes at MR imaging in the aging brain: data from the Cardiovascular Health Study. Radiology 1997;202:33-9.

[33] Scheltens P, Leys D, Barkhof F, Huglo D, Weinstein HC, Vermersch P, et al. Atrophy of medial temporal lobes on MRI in "probable" Alzheimer's disease and normal ageing: diagnostic value and neuropsychological correlates. J Neurol Neurosurg Psychiatry 1992;55:967-72.

[34] Frisoni GB, Geroldi C, Beltramello A, Bianchetti A, Binetti G, Bordiga $\mathrm{G}$, et al. Radial width of the temporal horn: a sensitive measure in Alzheimer disease. AJNR Am J Neuroradiol 2002;23:35-47.

[35] Wahlund LO, Barkhof F, Fazekas F, Bronge L, Augustin M, Sjogren M, et al. C. European Task Force on Age-Related White Matter. A new rating scale for age-related white matter changes applicable to MRI and CT. Stroke 2001;32:1318-22.

[36] Talairach J, Tournoux P. Co-Planar Sterotaxic Atlas of the Human Brain: 3-Dimensional Proportional System - an Approach to Cerebral Imaging. New York: Thieme Medical Publishers; 1988.

[37] Wallin A, Kapaki E, Boban M, Engelborghs S, Hermann DM, Huisa B, et al. Biochemical markers in vascular cognitive impairment associated with subcortical small vessel disease - A consensus report. BMC Neurol 2017;17:102.

[38] Wardlaw JM, Smith EE, Biessels GJ, Cordonnier C, Fazekas F, Frayne R, S.T.f.R.V.c.o. nEuroimaging, et al. Neuroimaging standards for research into small vessel disease and its contribution to ageing and neurodegeneration. Lancet Neurol 2013;12:822-38.

[39] Delbecq AL, Van de Ven AH, Gustafson DH. Group techniques for program planning. Glenview, IL: Scott, Foresman, and Co; 1975.

[40] Salvadori E, Poggesi A, Valenti R, Pracucci G, Pescini F, Pasi M, et al. Operationalizing mild cognitive impairment criteria in small vessel disease: the VMCI-Tuscany Study. Alzheimers Dement 2016; 12:407-18

[41] Folstein MF, Folstein SE, McHugh PR. "Mini-mental state". A practical method for grading the cognitive state of patients for the clinician. J Psychiatr Res 1975;12:189-98.

[42] Nasreddine ZS, Phillips NA, Bedirian V, Charbonneau S, Whitehead V, Collin I, et al. The Montreal Cognitive Assessment, MoCA: a brief screening tool for mild cognitive impairment. J Am Geriatr Soc 2005;53:695-9.

[43] Dong Y, Sharma VK, Chan BP, Venketasubramanian N, Teoh HL, Seet RC, et al. The Montreal Cognitive Assessment (MoCA) is superior to the Mini-Mental State Examination (MMSE) for the detection of vascular cognitive impairment after acute stroke. J Neurol Sci 2010; 299:15-8.

[44] Dong Y, Venketasubramanian N, Chan BP, Sharma VK, Slavin MJ, Collinson SL, et al. Brief screening tests during acute admission in patients with mild stroke are predictive of vascular cognitive impairment 3-6 months after stroke. J Neurol Neurosurg Psychiatry 2012; 83:580-5.

[45] Wallin A, Nordlund A, Jonsson M, Blennow K, Zetterberg H, Ohrfelt A, et al. Alzheimer's disease-subcortical vascular disease spectrum in a hospital-based setting: Overview of results from the Gothenburg MCI and dementia studies. J Cereb Blood Flow Metab 2016;36:95-113. 
[46] Hosseini AA, Meng D, Simpson RJ, Auer DP. Mesiotemporal atrophy and hippocampal diffusivity distinguish amnestic from non-amnestic vascular cognitive impairment. Eur J Neurol 2017; 24:902-11.

[47] B. Yew. Nation DAI. for the Alzheimer's Disease Neuroimaging. Cerebrovascular resistance: effects on cognitive decline, cortical atrophy, and progression to dementia. Brain 2017; 140:1987-2001.

[48] Chao LL, Buckley ST, Kornak J, Schuff N, Madison C, Yaffe K, et al. ASL perfusion MRI predicts cognitive decline and conversion from MCI to dementia. Alzheimer Dis Assoc Disord 2010; 24:19-27.

[49] Grau-Olivares M, Arboix A, Junque C, Arenaza-Urquijo EM, Rovira M, Bartres-Faz D. Progressive gray matter atrophy in lacunar patients with vascular mild cognitive impairment. Cerebrovasc Dis 2010;30:157-66.
[50] Nestor SM, Misic B, Ramirez J, Zhao J, Graham SJ, Verhoeff N, et al Small vessel disease is linked to disrupted structural network covariance in Alzheimer's disease. Alzheimers Dement 2017;13:749-60.

[51] Skrobot OA, Attems J, Esiri M, Hortobagyi T, Ironside JW, Kalaria RN, et al. Vascular cognitive impairment neuropathology guidelines (VCING): the contribution of cerebrovascular pathology to cognitive impairment. Brain 2016;139:2957-69.

[52] Hainsworth AH, Allan SM, Boltze J, Cunningham C, Farris C, Head E, et al. Translational models for vascular cognitive impairment: a review including larger species. BMC Med 2017;15:16.

[53] Lanza G, Bramanti P, Cantone M, Pennisi M, Pennisi G, Bella R. Vascular Cognitive Impairment through the Looking Glass of Transcranial Magnetic Stimulation. Behav Neurol 2017;2017:1421326.

[54] Bordet R, Ihl R, Korczyn AD, Lanza G, Jansa J, Hoerr R, et al. Towards the concept of disease-modifier in post-stroke or vascular cognitive impairment: a consensus report. BMC Med 2017;15:107.

\section{Did you know?}

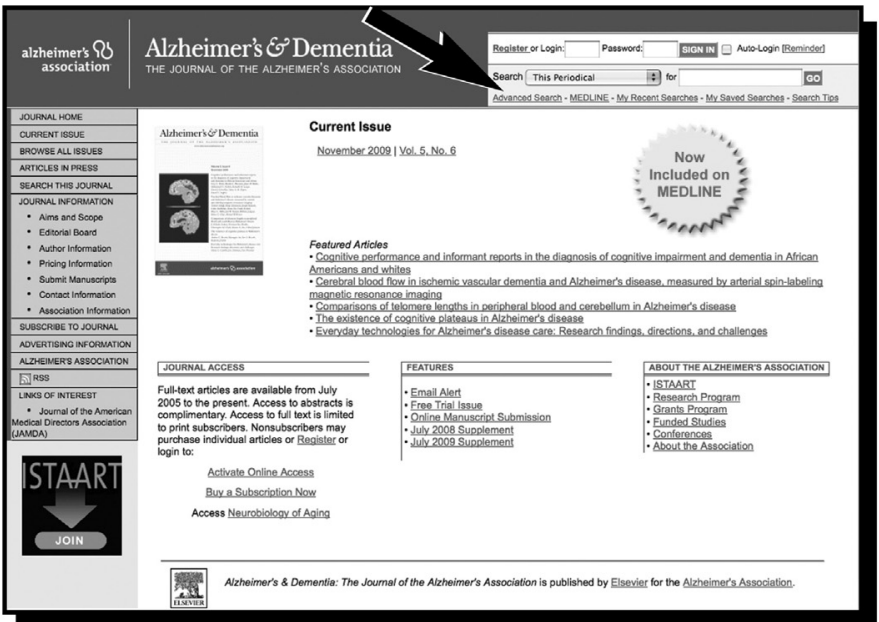

You can search

Alzheimer's

\& Dementia and

$500+$ top medical

and health

sciences journals

online, including

MEDLINE.

Visit www.alzheimersanddementia.org today! 